\title{
Diseño de un modelo de control de mantenimiento aeronáutico con base en la norma NTCGP 1000:2004, para realizar auditorías de gestión en la FAC
}

\section{CT. William Alexander Méndez Perdomo \\ TE. Miguel Andrés Bolivar Parsons}

\section{Descripción}

Esta investigación pretende formular una alternativa acorde con las orientaciones de la Fuerza Aérea Colombiana para cumplir con el plan institucional 2006-2019 a través de la Norma Técnica Colombiana en la Gestión Pública NTC GP1000: 2004. Asimismo, se tomaron como referencia formatos comerciales que permitieron a las diferentes entidades públicas y privadas realizar una constante revisión de los procesos internos y externos cuya finalidad consiste en buscar un mejoramiento continuo, teniendo en cuenta las normas de calidad internacional que buscan establecer una estandarización en beneficio de los usuarios.

\section{Objetivos}

\section{Objetivo general}

Diseñar un modelo para el control de contratistas en mantenimiento aeronáutico para la Fuerza Aérea Colombiana, con base en la Norma Técnica Colombiana en la Gestión Pública NTC GP1000: 2004, con el fin de realizar auditorías de gestión en mantenimiento aeronáutico.

\section{Objetivos especificos}

- Revisar la normatividad vigente en la Fuerza Aérea Colombiana para auditorías a empresas prestadoras de servicios de mantenimiento aeronáutico.

- Comparar los modelos aplicados a los contratistas de otros sectores económicos y verificar si cumple con los estándares de gestión que permitan auditar el mantenimiento aeronáutico.

- Evaluar el modelo de control de la Norma Técnica Colombiana en la Gestión Pública NTC GP1000: 2004, con base de que sea adecuado, necesario, suficiente y viable para la Fuerza Aérea Colombiana.

- Diseñar un modelo de control que permita realizar auditorías a los proveedores de servicios de mantenimiento aeronáutico de acuerdo con la Norma Técnica Colombiana en la Gestión Pública NTC GP1000: 2004

\section{Metodología}

Se aplicó la investigación documental y evaluativa con énfasis en la revisión bibliográfica especializada, ya que se 
estudió la Norma Técnica Colombiana en la Gestión Pública NTC GP1000:2004 para conocer como se manejan los estándares de calidad y asi obtener los conceptos y términos de referencias necesarios para dar respuesta al objeto de esta investigación, de igual manera se complementó con una investigación cualitativa para establecer el diagnóstico del estado actual en los procesos de auditoria con los que cuenta la Fuerza Aérea Colombiana enfocado a las empresas prestadoras de servicios aeronáuticos

\section{Resultados}

La Fuerza Aérea establecerá actividades de auditorías planeadas, orientadas a verificar actividades y los resultados de los mantenimientos efectuados por terceros, buscando aumentar el nivel de confiabilidad de estos trabajos para minimizar los posibles errores, evitando futuros accidentes.

Una organización que necesite realizar auditorías deberá implementar y gestionar un programa efectivo de auditoria, cuyo propósito es planear el tipo y el número de auditorias, e identificar y suministrar los recursos necesarios para realizarlas teniendo en cuenta los parámetros de la norma ISO 19011. De ahi que se pueda aplicar el ciclo PHVA para las auditorias, enfocándolas en el mantenimiento. Estas auditorias aplican para todos los proveedores externos de mantenimiento que cumplan con todo el proceso contractual y vayan a iniciar la fase de ejecución. Asi mismo, se anexan todos los formatos de auditorias externas a los proveedores de servicios en mantenimiento para la Fuerza Aèrea colombiana, los mapas de procesos, y la caracterización de auditores externos.

\section{Bibliografia}

\section{FUERZA AÉREA COLOMBIANA PLAN ESTRATÉGICO} INSTITUCIONAL 2007-2019.

LEY 872 DE 2003 Por la cual se crea el sistema de gestión de la calidad en la Rama Ejecutiva del Poder Público y en otras entidades prestadoras de servicios.

NTC GP 1000:2004 Sistema de Gestión de la Calidad del sector Público.

NTC ISO/ IEC 17025 Requisitos generales para la competencia de los laboratorios de ensayo y calibración.

NTC ISO 9000 Sistema de Gestión de la Calidad. Fundamentos y Vocabulario.

NTC ISO 9001:2000 Sistema de Gestion de la Calidad.

NTC ISO 19011:2002 Directrices para la auditoria de los sistemas de gestión de la calidad y/o ambiental.

NTC OHSAS 18001:2000 Sistema de Gestión en Seguridad y Salud Ocupacional.

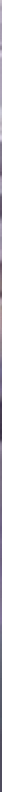

